\title{
A near/mid infrared search for ultra-bright submillimetre galaxies: Searching for Cosmic Eyelash Analogues
}

\author{
S. Iglesias-Groth ${ }^{1,2 \star}$, A. Díaz-Sánchez ${ }^{3}$, R. Rebolo ${ }^{1,2}$ and H. Dannerbauer ${ }^{1,2 \star} \dagger$ \\ 1 Instituto de Astrofísica de Canarias, La Laguna 38200, Spain \\ ${ }^{2}$ Departamento de Astrofísica de la Universiad de La Laguna, Avda. Francisco Sánchez, La Laguna, 38200, Spain \\ ${ }^{3}$ Departamento Física Aplicada, Universidad Politécnica de Cartagena, Campus Muralla del Mar, 30202 Cartagena, Murcia, Spain
}

\begin{abstract}
We present results from a near/mid IR search for submillimetre galaxies over a region of $6230 \mathrm{sq}$ deg. of the southern sky. We used a cross-correlation of the VISTA Hemispheric Survey (VHS) and the WISE database to identify bright galaxies $\left(\mathrm{K}_{s} \leqslant\right.$ 18.2) with near/mid IR colours similar to those of the high redshift lensed sub-mm galaxy SMM J2135-0102. We find 7 galaxies which fulfill all five adopted near $/ \mathrm{mid}$ IR colour (NMIRQC) criteria and resemble the SED of the reference galaxy at these wavelengths. For these galaxies, which are broadly distributed in the sky, we determined photometric redshifts in the range $\mathrm{z}=1.6-3.2$. We searched the VHS for clusters of galaxies, which may be acting as gravitational lenses, and found that 6 out of the 7 galaxies are located within 3.5 arcmin of a cluster/group of galaxies. Using the $\mathrm{J}_{-} \mathrm{K}_{s}$ vs $\mathrm{J}$ sequences we determine photometric redshifts for these clusters/groups in the range $z=0.2-0.9$. We propose the newly identified sources are ultra-bright high redshift lensed SMG candidates. Follow-up observations in the sub-mm and $\mathrm{mm}$ are key to determine the ultimate nature of these objects.
\end{abstract}

Key words: galaxies - submillimetre: galaxies.

\section{INTRODUCTION}

Since their discovery (Smail et al. 1997; Hughes et al. 1998) the so-called submillimetre galaxies (SMGs) are a vital galaxy population in order to understand the formation and evolution of massive galaxies in the distant universe (see for a review Casey et al. 2014). These systems have extreme star formation rates of several hundred to thousand solar masses per year (e.g., Magnelli et al. 2012), are molecular gas-rich with $\mathrm{M}_{\text {mol-gas }}=\sim$ few times $10^{10} \mathrm{M}_{\odot}$ (e.g., Greve et al. 2005; Bothwell et al. 2013) and the redshift distribution peaks $\approx z=2.2-3.0$ (Chapman et al. 2005; Simpson et al. 2014; Miettinen et al. 2015; Strandet et al. 2016) depending on the selected wavelength in the (sub)mm window. Typical sizes of these dusty starbursts range between $\mathrm{R}_{e}=0.6-2.0 \mathrm{kpc}$ (Ikarashi et al. 2015; Simpson et al. 2015; Hodge et al. 2016). Mergers and cold gas infall are potential agents of such star formation, however the available studies have not yet established the main cause of the high starformation rates (e.g., Swinbank et al. 2008; Hayward et al. 2013; Michalowski et al. 2012; Narayanan et al. 2015).

Despite intrinsic luminosities of $\mathrm{L}_{b o l} \geqslant$ few times $10^{12}$

* E-mail: sigroth@iac.es

$\dagger$ This paper makes use of data from catalogues VISTA and WISE to $10^{13} \mathrm{~L}_{\odot}$ (Magnelli et al. 2012; Ivison et al. 2013), these dusty galaxies are very challenging objects for observational studies in the near IR (e.g., Dannerbauer et al. 2002, 2008; Younger et al. 2007) and mid infrared (e.g. Pope et al. 2008). The brightest unlensed SMGs have observed flux densities of up to $\sim 10$ mJy at $850 \mu \mathrm{m}$ (e.g., Karim et al. 2013). Gravitational lensing via massive galaxy clusters can enhance the apparent brightness of SMGs without altering their colours (Smail et al. 1997) and cluster surveys (e.g. Smail et al. 2002; Johansson et al. 2011) indeed led to the detection of SMGs with high amplification factors (30-40) like that of the outstanding galaxy SMM J2135-0102 at $z=2.3259$ (the Cosmic Eyelash, hereafter SMM J2135, Swinbank et al. 2010; Ivison et al. 2010; Danielson et al. 2011). The South Pole Telescope and the Herschel and Planck space missions have unveiled similarly bright lensed SMGs (Negrello et al. 2010; Vieira et al. 2010, 2013; Weiss et al. 2013; Cañameras et al. 2015; Harrington et al. 2016; Strandet et al. 2016). In spite of the amplification factors provided by lensing, these SMGs are still rather faint in the optical and near-IR as a consequence of internal dust obscuration, and their properties in this spectral range are not well understood yet (e.g. Dannerbauer et al. 2002, 2004, 2008; Dunlop et al. 2004; Younger et al. 2007; Walter et al. 2012; Simpson et al. 2014; Hodge et al. 2015; Dye et al. 2015). The spectral energy distribu- 
tion of bright high redshift sources like SMM J2135-0102 (hereafter SMM J2135) shows a very steep increase in flux as we move from the optical to the near-IR and mid-IR which could potentially be used to identify other similar galaxies.

We took advantage of the VISTA Hemisphere Survey $(\mathrm{VHS})^{1}$ which has already covered an area of more than 8000 sq. deg. of the southern sky at near-IR bands and the Wide-field Infrared Survey Explorer (WISE) mission archive database in the mid-IR in order to find brighter analogues of SMM J2135. We adopted as a reference the near and midIR colours of this galaxy. Given the limiting magnitudes of the VHS/WISE surveys we expect the selected sample of similar colour galaxies will consist mainly of gravitationally lensed sources (Blain 1996, Negrello et al. 2007). Lensing of SMGs is strongly favored due to the steep number counts of this source population (Blain et al. 1996; Perrotta et al. 2002, 2003; Negrello et al. 2007; Negrello et al. 2010). Thus, if we conduct searches for sources with similar SEDs as known dusty starbursts in the near/mid IR wavelength regime, the above mentioned lensing bias should guarantee that the majority of the selected sources are massive galaxies with strong FIR/submm emission triggered by on-going intense star formation. Thus, the contamination by massive, early type galaxies should be rather negligible.

Our goal is to find the brightest high redshift $(\mathrm{z} \sim 2)$ analogues of SMM J2135 in the sky such that follow-up studies can be performed in the optical, near/mid IR with moderate investment of observing time at the largest telescopes. Finding new examples of this important class of strongly lensed star-forming galaxies should help to determine their extinction properties in the visible/IR, perform extensive characterisation in the millimetre/submillimetre range and obtain a better understanding of how and where stars are formed in these galaxies. Brighter analogues of the SMG SMM J2135 in the redshift range $\mathrm{z}=2-3$ will also allow us to investigate star formation on $100 \mathrm{pc}$ scales as a function of redshift using ALMA (Dye et al. 2015, Swinbank et al. 2015).

In the following sections we describe our search for bright SMGs in a large fraction of (approx. $30 \%$ ) of the southern sky and the resulting candidate galaxies. In Section 2 we present the infrared data from VISTA/VHS and WISE (Vega mag-system) used in the search, the photometry of the reference galaxy SMM J2135 and the adopted colour criteria to identify potential analogues of this galaxy. Section 3 reports the results of the search and the identification of clusters of galaxies close to the new candidate SMGs.

\section{THE SEARCH}

We will take advantage of the large sky coverage provided by the near-IR VISTA Hemispheric Survey (VHS) and the midIR WISE mission to conduct a search for bright analogues of the SMG SMM J2135.

\subsection{The VISTA Hemispheric Survey: VHS}

The VHS is a near-infrared ESO public survey designed to map the entire Southern hemisphere in the $\mathrm{J}$ and $\mathrm{K}_{s}$ broad band filters with average $5 \sigma$ depths of $\mathrm{J}=19.5 \pm$ $0.3 \mathrm{mag}$ and $\mathrm{K}_{s}=18.5 \pm 0.3 \mathrm{mag}$. In some particular areas also $\mathrm{Y}$ and $\mathrm{H}$ band observations are performed. The 4-m VISTA telescope (Emerson 2001; Emerson et al. 2004) operates since 2009 at ESO's Cerro Paranal Observatory in Chile and has so far covered a sky area of about $8000 \mathrm{deg}^{2}$. It is equipped with a wide-field infrared camera VIRCAM (Dalton et al. 2006) composed of 16 Raytheon detectors 20482048 pixel array each, with a mean plate scale of 0.34 arcsec, giving a field of view of 1.65 degrees in diameter.

The VHS images are processed and calibrated automatically by a dedicated science pipeline implemented by the Cambridge Astronomical Survey Unit (CASU). Standard reduction and processing steps include dark and sky subtraction, flat field correction, linearity correction, destripe and jitter stacking. For a detailed description we refer to the CASU webpage http://casu.ast.cam.ac.uk/surveysprojects/vista as well as to Irwin et al. (2004) and Lewis et al. (2010). The photometry provided in the VHS catalogue is calibrated using the magnitudes of colour-selected 2MASS stars converted onto the VISTA system using colour equations including terms accounting for interstellar reddening. We used the catalogue $\mathrm{J}$ and $\mathrm{K}_{s}$-band 1 arcsec aperture corrected (aperMag3) magnitudes for the selection of targets.

\subsection{The Wide-field Infrared Survey Explorer}

The NASA mission WISE completed an all-sky survey in four mid-IR bands (Wright et al. 2010) centred at 3.4, 4.6, 12 , and $22 \mu \mathrm{m}$ (referred as $\mathrm{W} 1, \mathrm{~W} 2$, W3 and $\mathrm{W} 4$, respectively) with an angular resolution of $6.1,6.4,6.5$, and 12.0 and $5 \sigma$ point source sensitivities of $0.07,0.1,0.86$ and 5.4 $\mathrm{mJy}$, respectively. The WISE photometric pipeline provides a number of quality flags useful in selecting point sources and extended sources. We use the AllWISE Source Catalog PSF profile-fit measurements that are made by chi-squared minimization on the "stack" of all Single-exposure frames in all bands covering a deep source detection ${ }^{2}$. Only measurements with $\mathrm{S} / \mathrm{N}>3.0$ will be considered.

\section{$2.3 \quad$ Reference galaxy}

As a reference for our search we adopted the near and midIR colours of SMM J2135. This extensively studied galaxy is lensed by a cluster of galaxies with no evidence for any intervening galaxy in the line of sight which could be affecting its near/mid-IR colours. SMM J2135 has an intrinsic restframe 8-1000 $\mu$ m luminosity, $\mathrm{L}_{b o l}$, of $2.3 \times 10^{12} \mathrm{~L}_{\odot}$ and a star formation rate estimated at $\sim 400 \mathrm{M}_{\odot} / \mathrm{yr}$ (Swinbank et al. 2010, Ivison et al. 2010). In the far-IR the SED can be described by dust components with temperatures of 30 and 60 K. A comparison with local ultra-luminous infrared galaxies like Arp 220 (Ivison et al. 2010) reveals that SMM J2135 

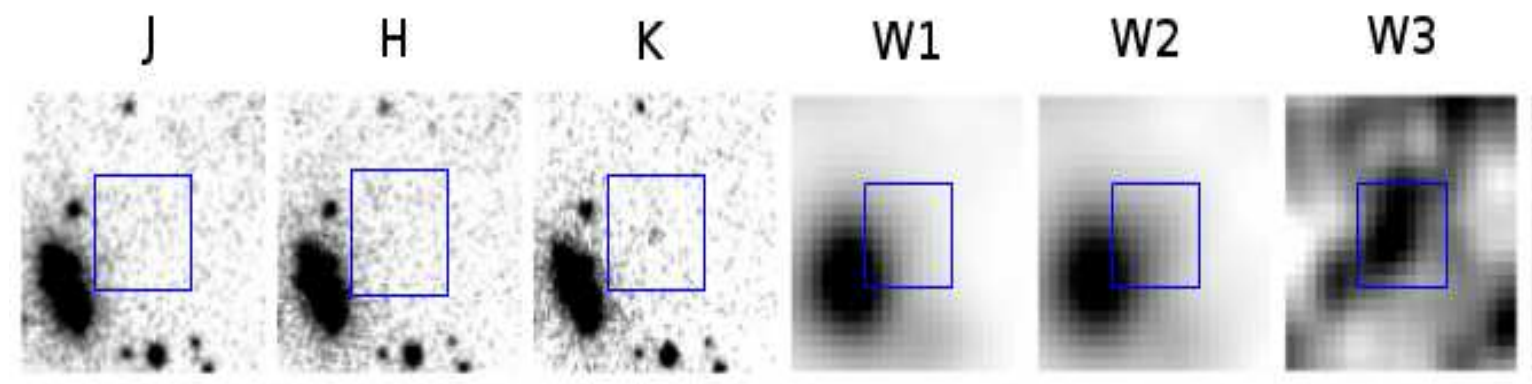

W4

Figure 1. Images $0.5 \times 0.5 \operatorname{arcmin}^{2}$ for SMM J2135-0102 in $\mathrm{J}, \mathrm{H}, \mathrm{K}_{s}$ (VISTA/VHS) and W1, W2, W3 and W4 (WISE) bands. The marked boxes are $12 \times 12 \operatorname{arcsec}^{2}$.

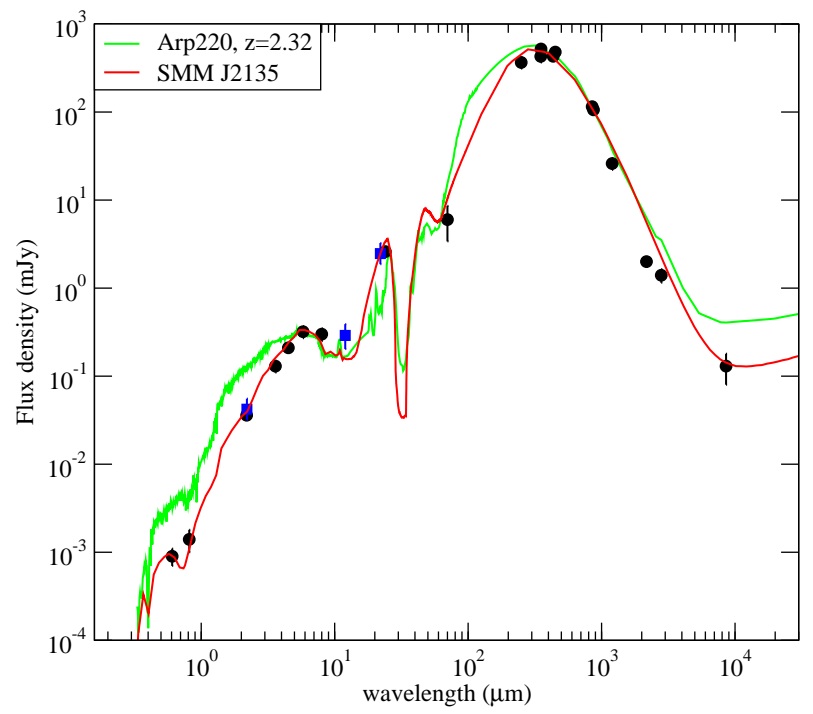

Figure 2. The Spectral Energy Distribution of SMM J2135-0102. The green line is the SED of the well known luminous IR galaxy Arp 220 redshifted to $\mathrm{z}=2.3$ with appropriate normalisation and the red line is the GRASIL SED given in Lapi et al. (2011). Black points from Ivison et al. (2010) and blue boxes are our measurements.

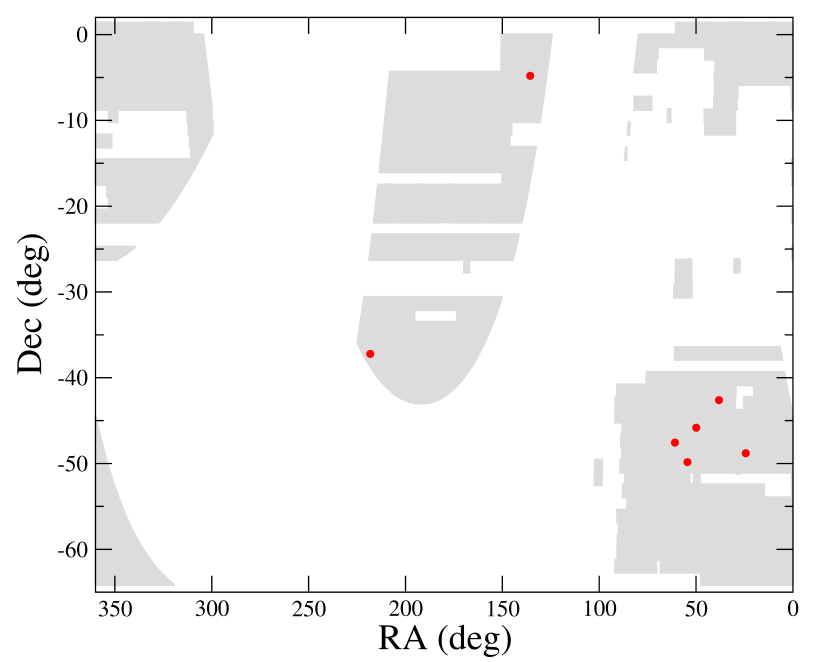

Figure 3. Positions of our 7 SMG candidates (red circles) and the sky area overlapped (gray) by the VHS/WISE survey. 
Table 1. Optical and near/mid-IR fluxes of the sub-mm galaxy SMM J2135-0102 ( $\mathrm{z}=2.3259)$

\begin{tabular}{|c|c|c|}
\hline Filter & Flux & Mag (Vega) \\
\hline$V$ & $0.9 \pm 0.2 \mu \mathrm{Jy}$ & $24.1 \pm 0.2$ \\
\hline$I$ & $1.4 \pm 0.4 \mu \mathrm{Jy}$ & $23.2 \pm 0.2$ \\
\hline$J^{1}$ & & $\gtrsim 20.5$ \\
\hline$K_{s}$ & $36 \pm 4 \mu \mathrm{Jy}$ & $18.00 \pm 0.07$ \\
\hline$[3.6]$ & $0.13 \pm 0.02 \mathrm{mJy}$ & $15.82 \pm 0.2$ \\
\hline$[4.5]$ & $0.21 \pm 0.02 \mathrm{mJy}$ & $14.83 \pm 0.1$ \\
\hline [8] & $0.32 \pm 0.05 \mathrm{mJy}$ & $13.2 \pm 0.2$ \\
\hline [24] & $2.6 \pm 0.2 \mathrm{mJy}$ & $8.6 \pm 0.1$ \\
\hline$W 1^{2}$ & $0.12 \pm 0.02 \mathrm{mJy}$ & $16.0 \pm 0.2$ \\
\hline$W 2^{3}$ & $0.23 \pm 0.02 \mathrm{mJy}$ & $14.7 \pm 0.1$ \\
\hline$W 3^{4}$ & $0.24 \pm 0.05 \mathrm{mJy}$ & $12.8 \pm 0.2$ \\
\hline$W 4$ & $2.5 \pm 0.8 \mathrm{mJy}$ & $8.8 \pm 0.3$ \\
\hline
\end{tabular}

2 Transformed from the Spitzer [3.6] band using the template SED of SMM J2135.

3 Transformed from the Spitzer [4.5] band using the template SED of SMM J2135.

4 Transformed from the Spitzer [8] band using the template SED of SMM J2135.

Table 2. Infrared colour criteria of potential SMM J2135 analogue galaxies

\begin{tabular}{ccc}
\hline Mag & Color & Mag. \\
\hline 2.0 & $<J-K_{s}$ \\
1.5 & $<K-W 1<$ \\
0.8 & $<W 1-W 2<$ & 2.5 \\
1.4 & $<W 2-W 3<$ & 1.8 \\
3.5 & $<W 3-W 4<$ & 4.5 \\
\hline
\end{tabular}

is slightly less luminous in the rest-frame optical to midIR (possibly due to stronger dust extinction) while rather similar in the $\mathrm{mm} / \mathrm{sub}-\mathrm{mm}$ range.

Fig. 1 shows VHS images in $\mathrm{J}, \mathrm{H}, \mathrm{K}_{s}$ and WISE images in the W1, W2, W3 and W4 bands of SMM J2135. No detection of this source was reported in the VHS and AllWISE catalogues, however after close inspection of the relevant images, SMM J2135 can be detected in the $\mathrm{K}_{s^{-}}$ band and in the W3 and W4 bands. Subsequently, we measured its magnitudes using aperture photometry and obtained $K_{s}=18 \pm 0.3, W 3=12.6 \pm 0.3$ and $W 4=8.8 \pm 0.3$ (Vega system). An upper limit on the J-band magnitude was also derived from the corresponding VHS image. The photometry was calibrated using nearby sources in the catalogue free of contaminants. In the H, W1 and W2 bands we achieve a marginal detection of SMM J2135 using the Laplacian of Gaussian filter, often called 'mexican hat', which helped to improve the detectability of sources in these filters. However, no reliable magnitudes could be determined from these images and the W1 and W2 magnitudes given in Table 1 were finally estimated converting the fluxes measured by Ivison et al in the [3.6] and [4.5] Spitzer bands, respectively. The final adopted fluxes and magnitudes of SMM J2135 are listed in Table 1.

The fluxes of SMM J2135 from the optical to centimetre wavelengths are plotted in Fig. 2 (black dots) in comparison with its SED as modeled by GRASIL from Lapi et al. (2011) and the SED of Arp 220 redshifted to $z=2.32$ with appropriate normalisation. The blue squares indicate our measurements in the $\mathrm{K}_{s}, \mathrm{~W} 3$ and $\mathrm{W} 4$ bands.

\subsection{Search method}

In order to find bright analogues of SMM J2135 we have correlated the AllWISE Source Catalogue with about $8000 \mathrm{deg}^{2}$ available from the VHS catalogue. The search is restricted to galactic latitudes $|b| \geqslant 20^{\circ}$, reducing the surveyed area to 6230 sq. deg., we retain galaxies for which we have detections in all the WISE bands and identify counterparts in the VHS catalogue within 1 arcsec of the AllWISE targets. To avoid bad candidates due to Galactic extinction, the VHS and AllWISE magnitudes are corrected for Galactic extinction with the use of the Schlegel et al. (1998) maps and the $A_{\lambda} / E(B-V)$ coefficients taken from Cardelli et al. (1989). Then, we selected those galaxies with colours consistent with the colour ranges listed in Table 2, i.e. with colours within $\pm 0.5 \mathrm{mag}$ of the SMM J2135 colours. We also restricted the selection to objects with $\mathrm{S} / \mathrm{N} \geqslant 3$ in all the WISE bands. A total of 7 galaxies verified all these conditions.

Very recently, colour selection procedures have been refined successfully to find SMGs (see Chen et al. 2016 and references therein). These authors used the so-called OpticalIR Triple Colour (OIRTC) selection procedure. We can also adopt a similar approach, imposing just one limit for each single colour in order to select SMGs, i.e take away one of the limits in each of the colours listed in Table 2. When we adopt the colour limits of Table 3 we find the same candidates. Therefore we adopt for simplicity the colour criteria of Table 3 which we could call the NIR/MIR Quintuple Colour (NMIRQC) procedure.

In Table 4 we list photometry for the 7 resulting SMG candidates. Their sky positions are shown in figure 3. Images of these candidates extracted from the surveys VHS and WISE are plotted in Fig. 4.

All our candidate sub-mm galaxies have well measured positions in the NIR images (accuracy better than $0.3^{\prime \prime}$ ) and are the only likely counterpart of the WISE sources. Only candidate 3 appears to be double in the $\mathrm{J}$ and $\mathrm{K}_{s}$ images. We note that this NIR/MIR selection could be advantageous with respect to other techniques in the sub-mm range which 
Table 3. Infrared single colour cuts to select sub-mm galaxies at high redshift

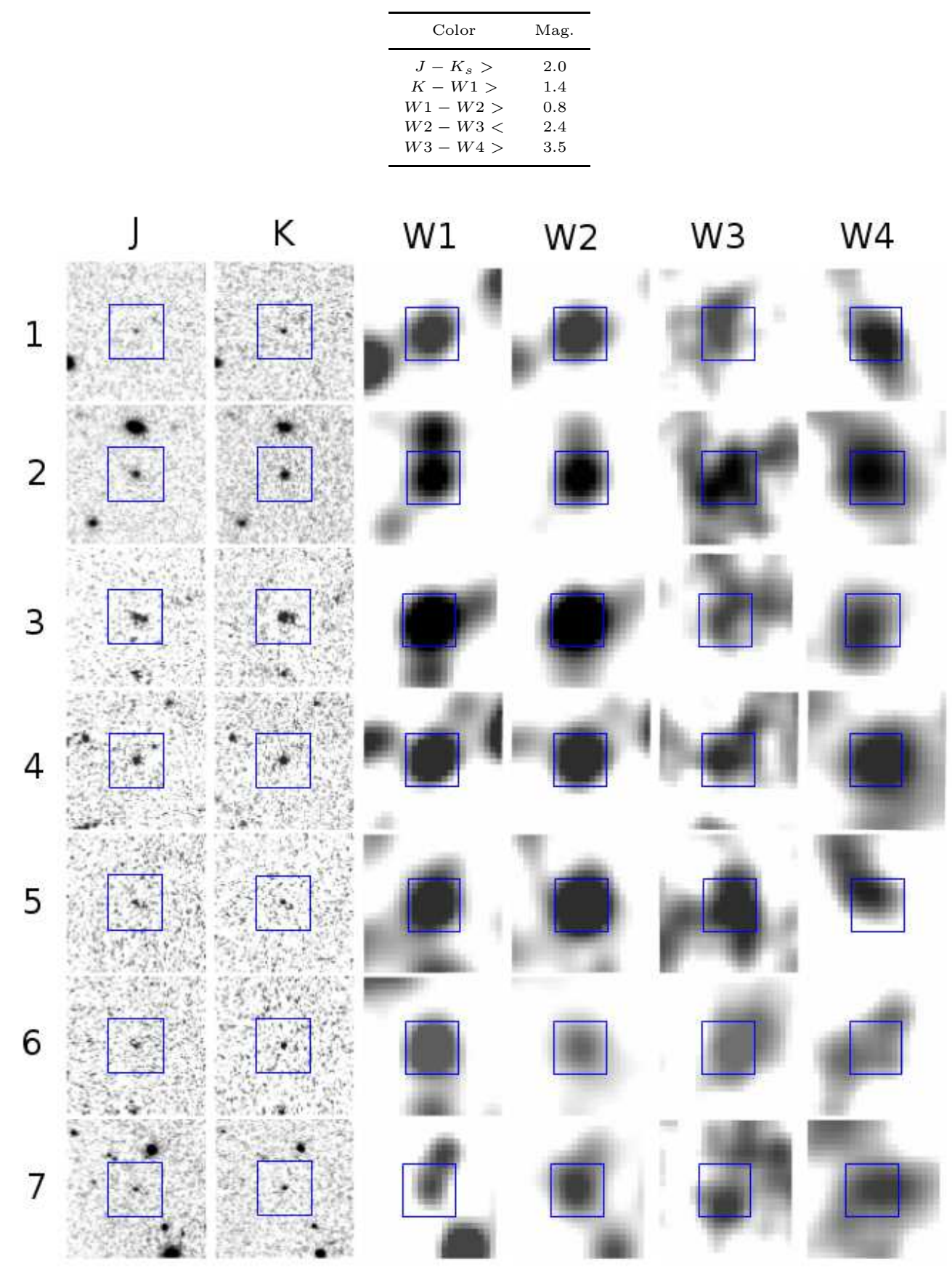

Figure 4. Images showing $0.5 \times 0.5 \operatorname{arcmin}^{2}$ centred around candidates in $\mathrm{J}, \mathrm{K}_{s}, \mathrm{~W} 1, \mathrm{~W} 2, \mathrm{~W} 3$ and $\mathrm{W} 4$ bands. The marked boxes are $12 \times 12 \operatorname{arcsec}^{2}$.

usually require follow-up interferometry to determine subarcsec position of the candidate galaxies.

\section{RESULTS AND DISCUSSION}

The 7 candidates that fulfill the colour criteria of the lensed sub-mm galaxy SMM J2135 present $J$-band magnitudes in the range 18.8-20.4 and $K_{s}$-band magnitudes in the range 16.8-18.2. The candidates are well identified in all bands.
In spite of the different spatial resolution of the VHS and WISE, there is good agreement in the positions of the identified sources.

In Figure 5 we plot colour-colour diagrams for the 7 candidates, SMM J2135, three SPT (South Pole Telescope) sources we have detected in VHS/WISE and one additional SMG lensed by a galaxy cluster reported by Gonzalez et al. 2011 (in this last case we have transformed Spitzer magnitudes to WISE magnitudes and there is a lower limit to the 

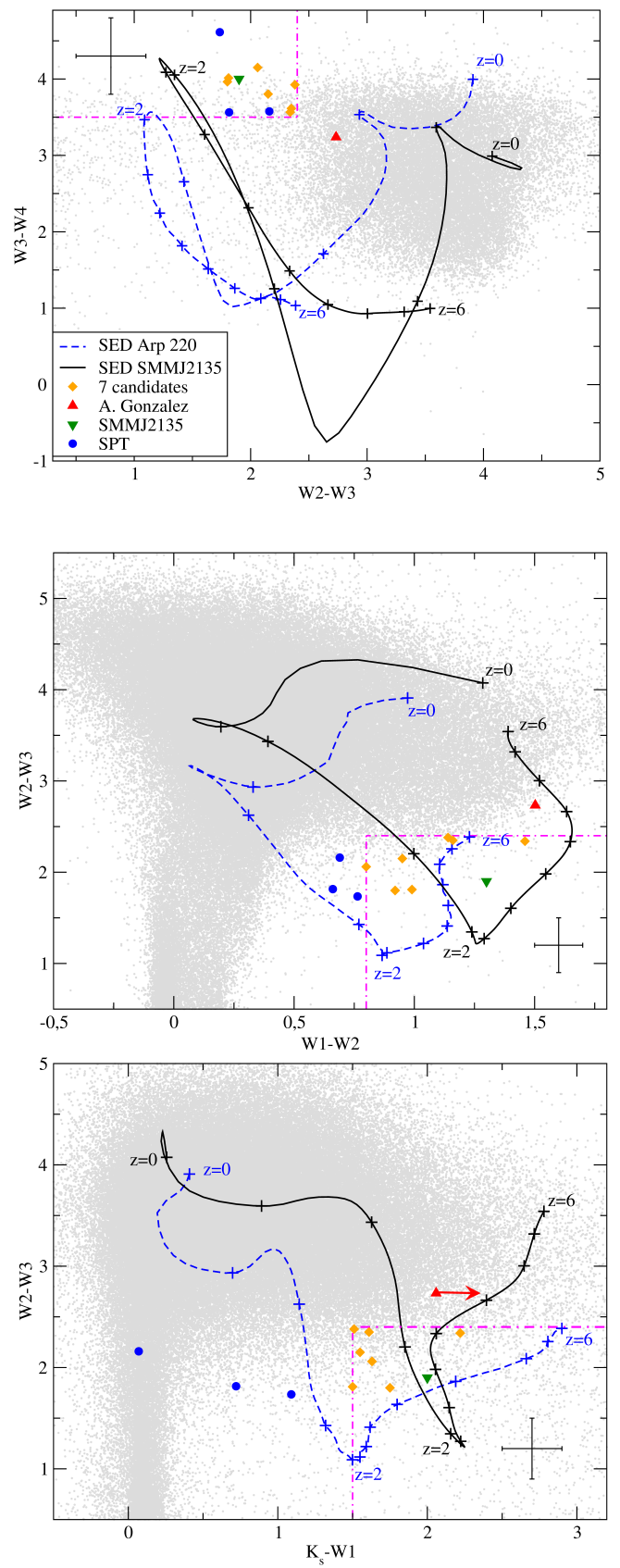

Figure 5. Colour-colour diagrams for our 7 candidates, SMM J2135, three SPT (South Pole Telescope) sources with redshift $2.23,2.51$ and 2.78 , and $\mathrm{a} z=2.79 \mathrm{SMG}$ lensed by a galaxy cluster given in Gonzalez et al. (2011). The grey points are field sources. The colour cuts in Table 3 are given by point-dashed lines and typical error bars are shown in each diagram. We have used continuous lines to show the position of the SMM J2135 GRASIL SED and Arp220 SED as a function of redshift (indicated by a number, crosses are drawn in the curves for each 0.5 increment of redshift).
$\mathrm{K}_{s}$ magnitude). The grey points are field sources. The colour cuts in Table 3 are given by point-dashed lines and typical error bars are shown in each diagram. We have also represented with continuous curves the SMM J2135 GRASIL SED and the Arp220 SED as a function of redshift (redshift is indicated by a number and crosses are drawn in the curves for each 0.5 increment of redshift). The three SPT sources have redshift 2.23, 2.51 and 2.78, they are lensed by foreground galaxies. While their WISE colours fall into the colour ranges we have adopted for SMGs, in the VHS bands there is contamination by the intervening lensing galaxy and the $\mathrm{K}_{s}$-W1 colours lie out of the adopted range. The colours of SMM J2135 $(\mathrm{z}=2.32)$ and the SMG identified by Gonzalez et al. $2011(\mathrm{z}=2.79)$ do probably represent the true colours of the original lensed galaxy because they are lensed by galaxy clusters and therefore photometric contamination in the near-IR bands is not expected. From these diagrams we can see that the redshift of our selected sources are likely between $\mathrm{z} \sim 1.6$ and $\mathrm{z} \sim 3.2$. Also, it is shown that the contamination of our sample by field sources is not large given the distant location of the adopted colour cuts with respect the bulk of the field sources. The SMM J2135 GRASIL SED does not match well the W3 band measurement, as we can see in figure 2, and colour differences between the SED and SMM J2135 come from this. Nevertheless the extreme values in colours W3-W4 and W2-W3 are obtained for redshift $\mathrm{z} \sim 2.5$, rather far from the colour-colour regions where most field sources are located.

In order to discuss the potential contamination of galaxies other than SMGs we have used the SWIRE template library (Polletta et al. 2007 ) which contains 25 templates including 3 ellipticals, 7 spirals, 6 starbursts (SB), 7 AGNs (3 type 1 AGNs, 4 type 2 AGNs), and 2 composite (starburst+AGN). The 3 ellipticals are 2, 5 and 13 Gyr old and the 7 spirals range from early to late types ( $\mathrm{S} 0-\mathrm{Sdm})$. Templates of moderately luminous AGN, representing Seyfert 1.8 and Seyfert 2 galaxies, were obtained by combining models, and spectra of a random sample of 28 Seyfert galaxies. The other six AGN templates include three templates representing optically-selected QSOs with different values of infrared/optical flux ratios and two type 2 QSOs. The composite $(\mathrm{AGN}+\mathrm{SB})$ templates are empirical templates, these objects contain a powerful starburst component, mainly responsible for their large infrared luminosities and an AGN component that contributes to the mid-IR luminosities.

In Figure 6 we can see that only spiral galaxies are located in the selection region in the $W 3-W 4$ vs. $W 2-$ $W 3$ colour-colour diagram at redshift $z \sim 2$ (top panel) but in the $W 3-W 2$ vs. $K_{s}-W 1$ diagram (central panel) these galaxies only go into the selection region for $z \sim 4$. So they do not fulfill simultaneously both colour conditions at the same redshift and therefore are not a likely source of contamination. The bottom panel of figure 6 shows the $W 3-W 4$ vs. $W 2-W 3$ colour-colour diagram for the 7 AGNs and the 2 composite $\mathrm{SB}+\mathrm{AGN}$ galaxies, none of them are in the selection region. The SED of SB galaxies is not too different from that of Arp220 and SMM J2135 and indeed these galaxies may enter the selection region for $1.7 \lesssim z \lesssim 3$.

In Tables 4 and 5 we complement the photometry of several SMG candidates with VHS measurements available in the $\mathrm{H}$ band and in the optical bands using CFHTLS. We have used redshifted GRASIL SEDs of SMM J2135 to 
determine the photometric redshift of our candidates, from $\mathrm{J}, \mathrm{H}, \mathrm{K}_{s}, \mathrm{~W} 1, \mathrm{~W} 2, \mathrm{~W} 3, \mathrm{~W} 4$-band measurements (H-band when available) using a standard $\chi^{2}$ minimization procedure. The results are given in Table 4 . All derived redshifts lie in the range $\mathrm{z}=1.6-3.2$ as expected. We find 2 galaxies with $\mathrm{K}_{s}$ $\leqslant 16.8,3$ galaxies in the range $\mathrm{K}_{s}=17-17.2$ and 2 galaxies with $\mathrm{K}_{s}=17.5$-18.2 Only 1 galaxy has photometric redshifts higher than $\mathrm{z}=3$ in our sample. In general our selected candidates are about 1-2 magnitudes brighter than typical lensed SMGs selected from Herschel surveys (e.g. Ma et al. 2015a; Calanog et al. 2014) or the SPT survey (Ma et al. 2015b). However, one source, HATLAS J142935.3-002836 alias G15v2.19, at $\mathrm{z}=1.027$ has similar NIR properties as our brightest candidates (Messias et al. 2014; Calanog et al. 2014; Ma et al. 2014).

In Fig. 7 we plot the SEDs of the candidate SMGs in comparison with the GRASIL SED of SMM J2135 shifted to the photometric redshift determined for each candidate. We note that in most cases the model fits very well all the nearIR and mid-IR bands. In the case of candidate 6 (VHSJ09020448), however, the fluxes deviate from the model at the shorter wavelength bands, probably because of the presence of an intervening galaxy in the line of sight which however is not detected in the available $\mathrm{J}$ and $\mathrm{K}_{s}$ images. From these fits we can estimate peak sub-mm fluxes above $\sim 500$ micron and a flux density at $1.4 \mathrm{~mm}$ above $\sim 15 \mathrm{mJy}$ for all of our targets. Very few sources are expected to be intrinsically bright enough to exceed these fluxes (Karim et al. 2013) and therefore we may expect that our sample is conformed by gravitationally lensed galaxies.

Weiss et al. (2013) measured the redshift distribution of 26 strongly lensed sources selected from the 2500 squaredegrees of the South Pole Telescope (SPT) survey with fluxes at $1.4 \mathrm{~mm}$ above $20 \mathrm{mJy}$. They found a redshift distribution of the SPT sample with a mean of $\mathrm{z}=2.0$, only 1 out of their 26 targets had redshift in the range $\mathrm{z}=2.5-3.2$. Our targets have redshifts consistent with the SPT distribution, but only for 5 targets we estimate $1.4 \mathrm{~mm}$ fluxes above 20 mJy from the fits plotted in Fig. 7. The surface density we infer for SMG sources selected in the near/mid IR to match $1.4 \mathrm{~mm}$ fluxes above $20 \mathrm{mJy}$ would be $5 / 6230 \mathrm{deg}^{-1}$ i.e. approximately 10 times less surface density that that found by Weiss et al. (2013) for potentially equivalent objects. We argue that this difference can be due to our selection criteria mostly identifying galaxies which are lensed by clusters of galaxies. Galaxies amplified by an intervening galaxy in the line of sight are likely to have photometric magnitudes in the $\mathrm{J}$ and $\mathrm{K}_{s}$ bands affected by the lens and may not verify our selection criteria. Only a few cases in SPT are cluster lensing galaxies. Spilker et al. (2016) find that 4 of 47 SPT sources are lensed by clusters. The NMIRQC procedure, proposed here, could be a very effective tool to identify strongly lensed SMGs by clusters of galaxies. SMGs detected by Herschel have $500 \mu \mathrm{m}$ fluxes in the range 100-350 mJy (Bussmann et al. 2013). For our candidates, we estimated $500 \mu \mathrm{m}$ fluxes from the fitted SEDs, the results range from 160 to 980 mJy and are listed in Table 4. Follow-up sub$\mathrm{mm} / \mathrm{mm}$ observations of the candidates will demonstrate if indeed we are dealing with strongly lensed SMGs.

From the same SEDs we obtained 60 and $100 \mu \mathrm{m}$ fluxes which used with the FIR/radio relation by Condon et al. (1992) allowed us to predict radio emission at $1.4 \mathrm{GHz}$ in the range 0.09-0.36 mJy. To our knowledge no detection of radio emission has been reported in the literature for our targets.

\subsection{Clusters of galaxies and sub-mm galaxy candidates}

Using the red sequence method (Gladders et al. 2000) and the VHS survey we have searched for clusters of galaxies near our SMG candidates and identified several galaxy clusters in the range $0.2<z<0.9$.

We searched for local over densities of galaxies using the $J-K_{s}$ versus $J$ colour-magnitude diagram. For these redshifts, the slope of the red-sequence in the diagram is nearly -0.03 (Cohn et al., 2007). We took $J-K_{s}$ colour slices of amplitude 0.2 magnitudes and searched for local overdensities in circular areas around the brightest cluster galaxy $(\mathrm{BCG})$ with radius $\sim 1 \mathrm{Mpc}$. We selected a cluster candidate if the density of galaxies of the cluster were larger than 3 times the mean density and had more than 10 galaxies in the red-sequence. We adopted as cluster radius the largest radius where the over density was detected.

In Figure 8 we can see the colour-magnitude diagrams for the six clusters of galaxies we have detected near SMG candidates $1,2,4,5,6$, and 7 , respectively. The $J-K_{s}$ colour of the BCG is an indicator of the redshift of the cluster (Tonini et al 2012). Only for candidate 3 we do not find a nearby cluster. Indeed this candidate is the only one that appears to be double in the VHS images of Figure 4, suggesting the presence of an intervening galaxy in the line of sight. We estimated the redshift of the clusters from the colours predicted by the red sequence fit (see Fig. 8) for each BCG. In Table 6 we present the main properties of all these clusters and group of galaxies, including reacheness and radius estimates. The VHS $J$-band images $10 \times 10 \operatorname{arcmin}^{2}$ are shown in Fig. 9, blue circles denote clusters and are plotted with the cluster radii given in Table 6 . The blue boxes mark the position of SMG candidates. Red circles denote the position of identified candidate galaxy members. Remarkablly candidates 1, 2 and 4 are located within clusters and candidate 5 is just at 1.8 arcmin from the centre of a cluster. The identification of clusters of galaxies within 3.5 arcmin of six out of 7 candidates further supports the suggestion that these objects are lensed SMGs.

\section{CONCLUSIONS}

We report results on a search for bright high redshift submillimetre galaxies , analogues of the lensed galaxy SMM J2135$0102, \mathrm{z}=2.3$, via a cross-correlation of the WISE and VHS databases performed over a high galactic latitude southern sky area of $6230 \mathrm{sq}$ deg. We adopted as reference the near/mid-IR colours of this galaxy and searched for galaxies of similar colours with reported detections in all $\mathrm{J}, \mathrm{K}_{s}$, $\mathrm{W} 1, \mathrm{~W} 2, \mathrm{~W} 3$ and $\mathrm{W} 4$ bands. We find 7 galaxies brighter than $\mathrm{K}_{s}=18.2$ matching the adopted colour criteria.

Using redshifted SEDs of the reference galaxy we determine the most likely redshift for each galaxy and find that our sample lie in the range $\mathrm{z}=1.6-3.2$. From the best individual fits we estimate sub-mm and mm fluxes for each target and conclude that 5 out of the 7 galaxies may have fluxes 

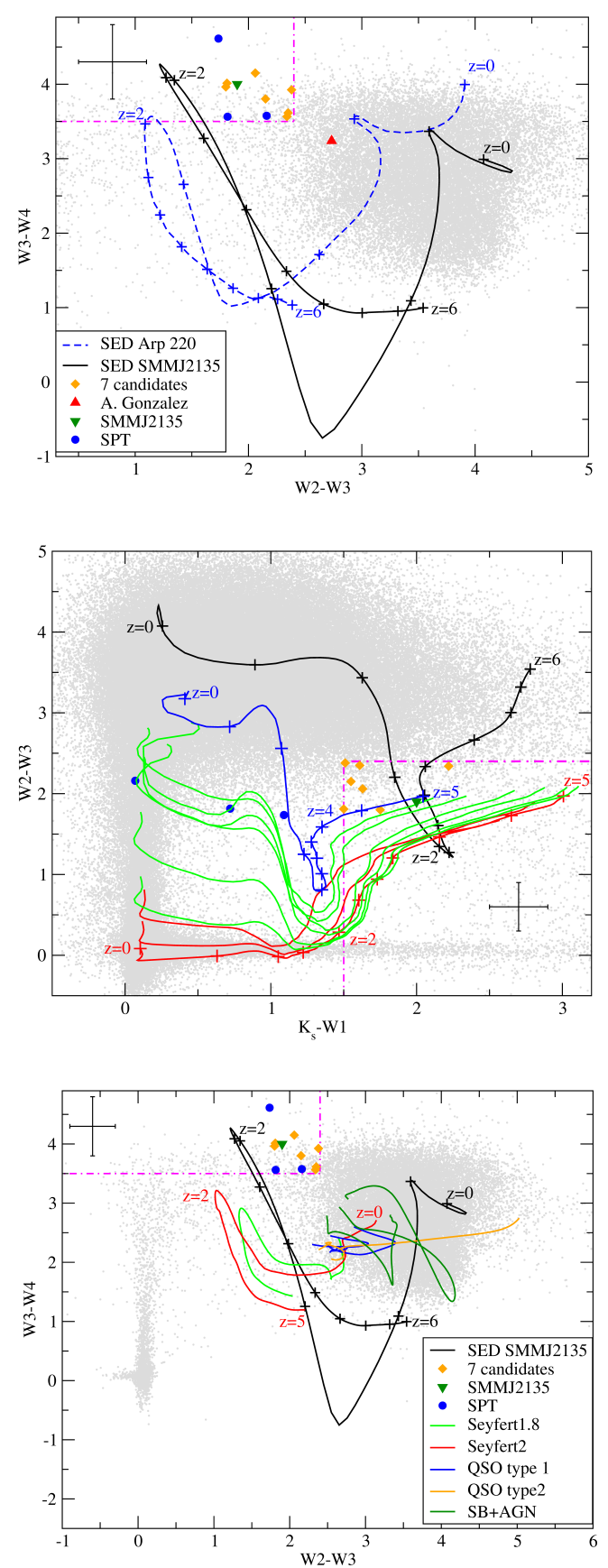

Figure 6. Colour-colour diagrams for our 7 candidates, SMM J2135 and three SPT (South Pole Telescope) sources with redshift 2.23, 2.51 and 2.78. The grey points are field sources. The color cuts in Table 3 are given by point-dashed lines and tipical error bars are shown in each diagram. Top and central panels: the continuous curves show the position of SMM J2135 GRASIL SED, 3 elliptical galaxies which are 2, 5 and $13 \mathrm{Gyr}$ old and 7 spirals range from early to late types (S0-Sdm). Bottom panel: the continuous curves show the position of SMM J2135 GRASIL SED, 2 moderately luminous AGN, representing Seyfert 1.8 and Seyfert 2 galaxies, 3 type 1 QSOs, 2 type 1 QSOs and 2 composite starburst(SB)+AGN galaxies (in some curves redshift is indicated by a number in the same colour and crosses are drawn in these curves for each 0.5 increment of redshift).

above $20 \mathrm{mJy}$ at $1.4 \mathrm{~mm}$. The surface density that we determine for lensed SMGs ( $\sim 1$ galaxy per 890 sq. deg.) is about 10 times lower than that obtained by Weiss et al. (2013) for $\mathrm{z}=1.6-3.2 \mathrm{sub}-\mathrm{mm}$ galaxies with $1.4 \mathrm{~mm}$ fluxes above $20 \mathrm{mJy}$. The difference can be due to our selection criteria mostly selecting SMGs amplified by clusters of galaxies, as those amplified by intervening galaxies may have their infrared colours affected.

Using VHS and the J-K vs J cluster sequence method, we have identified potential clusters/groups of galaxies near 6 of these candidates. Photometric redshifts locate most of these nearby clusters in the range $\mathrm{z}=0.2-0.9$.

We propose that our near/mid-IR selection procedure 


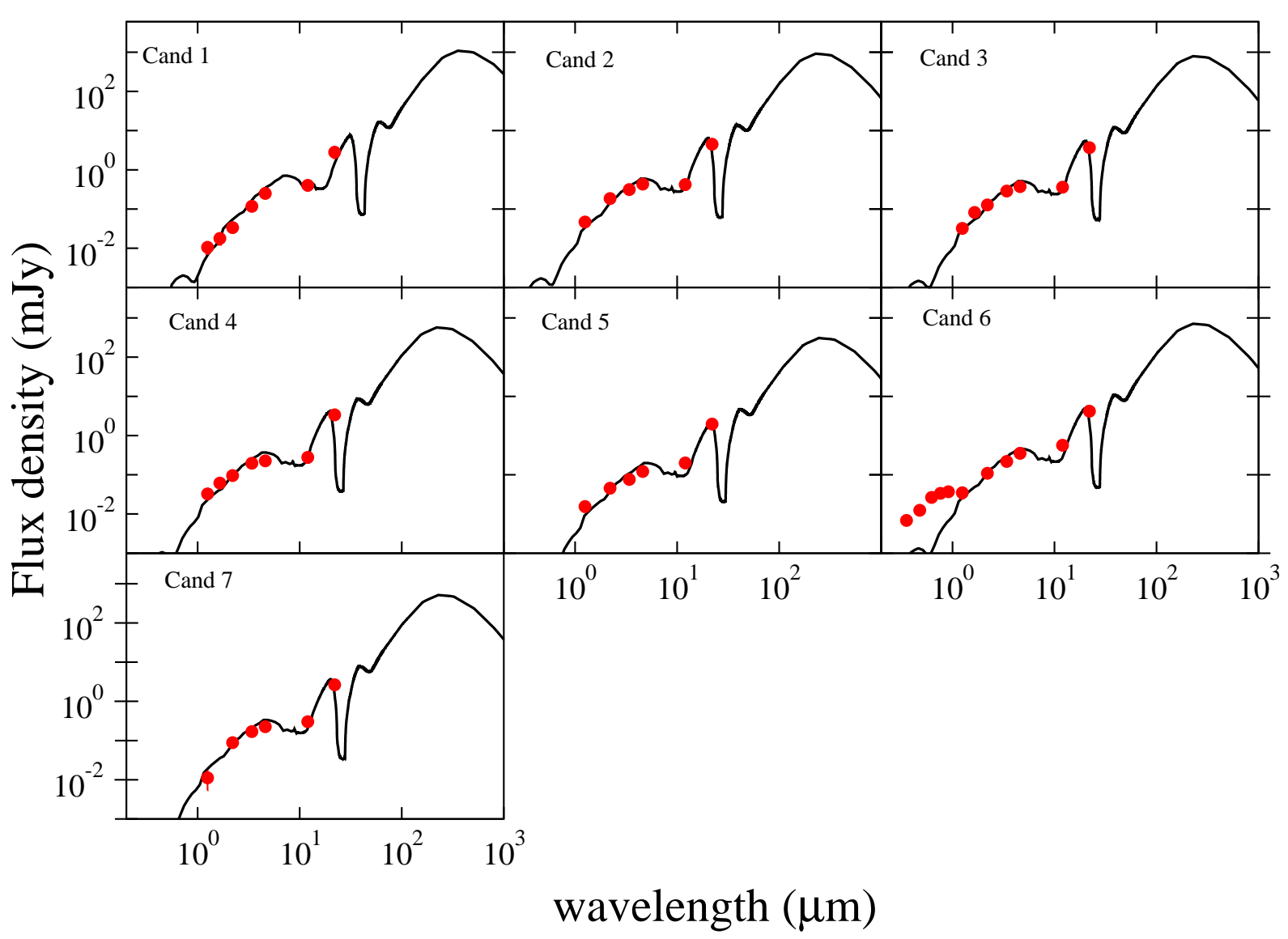

Figure 7. Fluxes of the SMG candidates (red points) in comparison with the SMM J2135 GRASIL SED shifted to the photometric redshift of each candidate (black lines). Note that only candidate ID1 has values greater than 500mJy (980mJy) at 500 microns. The rest have values between 160 and $430 \mathrm{mJy}$

Table 4. VISTA (http://horus.roe.ac.uk/vsa/index.html) and WISE magnitudes.

\begin{tabular}{|c|c|c|c|c|c|c|c|c|c|c|}
\hline ID & RA & Dec & $J$ & $H$ & $K_{s}$ & $W 1$ & $W 2$ & $W 3$ & $W 4$ & $z_{p h o t}$ \\
\hline 1 VHSJ0137-4848 & $01: 37: 24.39$ & $-48: 48: 25.1$ & $20.4 \pm 0.4$ & $19.4 \pm 0.4$ & $18.2 \pm 0.3$ & $16.03 \pm 0.05$ & $14.56 \pm 0.05$ & $12.2 \pm 0.3$ & $8.7 \pm 0.4$ & $3.2 \pm 0.2$ \\
\hline 2 VHSJ0232-4236 & $02: 32: 37.00$ & $-42: 36: 31.2$ & $18.8 \pm 0.2$ & & $16.5 \pm 0.1$ & $14.96 \pm 0.03$ & $13.97 \pm 0.04$ & $12.2 \pm 0.3$ & $8.2 \pm 0.3$ & $1.7 \pm 0.1$ \\
\hline 3 VHSJ0319-4549 & $03: 19: 38.74$ & $-45: 49: 40.2$ & $19.2 \pm 0.2$ & $17.7 \pm 0.2$ & $16.8 \pm 0.2$ & $15.06 \pm 0.03$ & $14.13 \pm 0.03$ & $12.3 \pm 0.3$ & $8.4 \pm 0.2$ & $1.7 \pm 0.1$ \\
\hline 4 VHSJ0337-4950 & $03: 37: 46.05$ & $-49: 50: 08.7$ & $19.2 \pm 0.2$ & $18.0 \pm 0.2$ & $17.1 \pm 0.2$ & $15.47 \pm 0.03$ & $14.68 \pm 0.04$ & $12.6 \pm 0.3$ & $8.5 \pm 0.2$ & $1.6 \pm 0.1$ \\
\hline 5 VHSJ0403-4733 & $04: 03: 39.52$ & $-47: 33: 37.4$ & $20.0 \pm 0.3$ & & $18.0 \pm 0.3$ & $16.49 \pm 0.05$ & $15.35 \pm 0.06$ & $13.0 \pm 0.3$ & $9.0 \pm 0.3$ & $1.9 \pm 0.1$ \\
\hline 6 VHSJ0902-0448 & $09: 02: 23.98$ & $-04: 48: 17.7$ & $19.1 \pm 0.3$ & & $17.0 \pm 0.2$ & $15.35 \pm 0.04$ & $14.20 \pm 0.05$ & $11.9 \pm 0.3$ & $8.2 \pm 0.3$ & $1.9 \pm 0.1$ \\
\hline 7 VHSJ1432-3713 & $14: 32: 41.33$ & $-37: 13: 40.6$ & $20.3 \pm 0.8$ & & $17.2 \pm 0.2$ & $15.63 \pm 0.05$ & $14.68 \pm 0.06$ & $12.5 \pm 0.3$ & $8.7 \pm 0.3$ & $1.7 \pm 0.1$ \\
\hline
\end{tabular}

can identify good candidates to bright high redshift lensed SMGs. Follow-up sub-mm observations (already on-going with APEX) and mm-observations (with e.g. LMT and ALMA) will reveal if indeed the selected objects are bright lensed SMGs and will provide accurate spectral redshifts. If confirmed, these would be excellent targets to carry out a systematic determination of the properties in the optical, near-IR, mid-IR, millimetre and submillimetre of SMGs in the redshift range 1.6-3.2.

The near/mid IR colour selection used in this work can potentially be extended in redshift space, particularly to higher redshifts where the redshift distribution of SMGs displays a peak (e.g. Bèthermin et al. 2015). Higher redshift searches increasingly demand higher sensitivity near-IR data. Future large scale deep near-IR surveys, as planned for the Euclid mission (ESA), in combination with WISE may enable the detection of a large population of bright lensed SMGs at various redshifts.

\section{ACKNOWLEDGEMENTS}

This work has been partially funded by projects "Participacion en el instrumento NISP y preparacion para la ciencia de EUCLID", ESP2015-69020-C2-1-R (MINECO). H.D. acknowledges financial support from the Spanish Ministry of Economy and Competitiveness (MINECO) under the 2014 Ramón y Cajal program MINECO RYC-2014-15686 


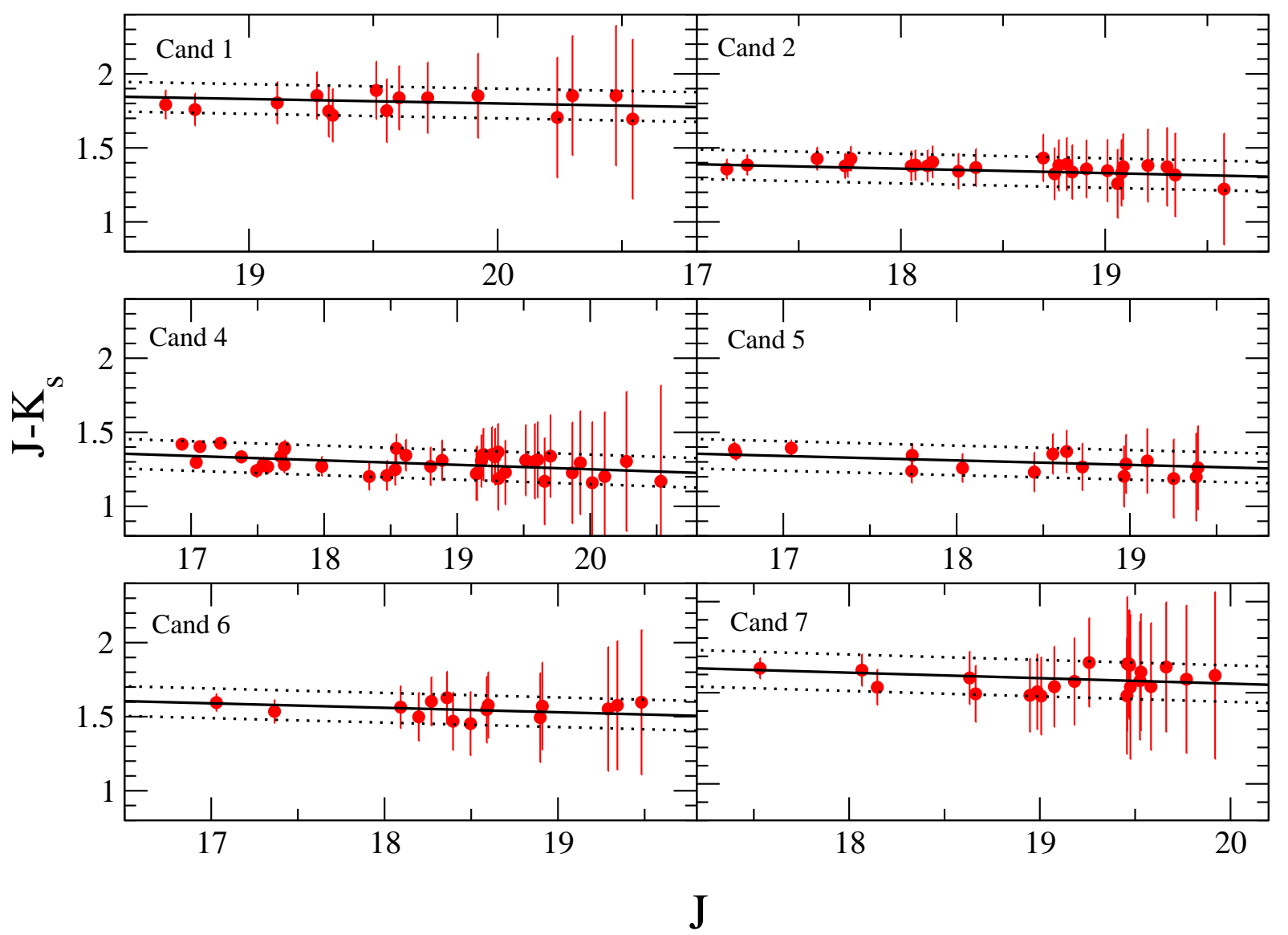

Figure 8. $J-K_{s}$ vs. $J$ colour-magnitude diagrams for 6 galaxy clusters near our SMG candidates (number labelled on each diagram). Diagrams show the objects within $1 \mathrm{Mpc}$ from the center of each galaxy cluster. The slope of the red sequence is -0.03 and the dotted lines are located at a separation of 0.1 magnitudes from the red sequence line.

Table 5. Optical magnitudes.

\begin{tabular}{ccccccc}
\hline ID & $u$ & $g$ & $r$ & $i$ & $z$ & Survey \\
& & & & & & \\
\hline 6 VHSJ0319-4549 & $21.81 \pm 0.01$ & $21.161 \pm 0.004$ & $20.341 \pm 0.003$ & $20.083 \pm 0.003$ & $19.984 \pm 0.006$ & CFHTLS \\
\hline
\end{tabular}

Table 6. Cluster of galaxies near some candidates

\begin{tabular}{|c|c|c|c|c|c|}
\hline Candidate & Redshift $^{1}$ & Galaxies $^{2}$ & Cluster radius ${ }^{3}$ & Distance $^{4}$ & Overdensity factor ${ }^{5}$ \\
\hline 1 VHSJ0137-4848 & 0.9 & 15 & 2.4 & 1.10 & 3.72 \\
\hline 2 VHSJ0232-4236 & 0.3 & 26 & 2.8 & 0.43 & 3.11 \\
\hline 4 VHSJ0337-4950 & 0.2 & 40 & 3.5 & 2.44 & 3.39 \\
\hline 5 VHSJ0403-4733 & 0.2 & 16 & 1.6 & 3.43 & 4.32 \\
\hline 6 VHSJ0902-0448 & 0.5 & 15 & 2.2 & 3.20 & 3.62 \\
\hline 7 VHSJ1432-3713 & 0.5 & 22 & 2.2 & 3.16 & 3.67 \\
\hline
\end{tabular}



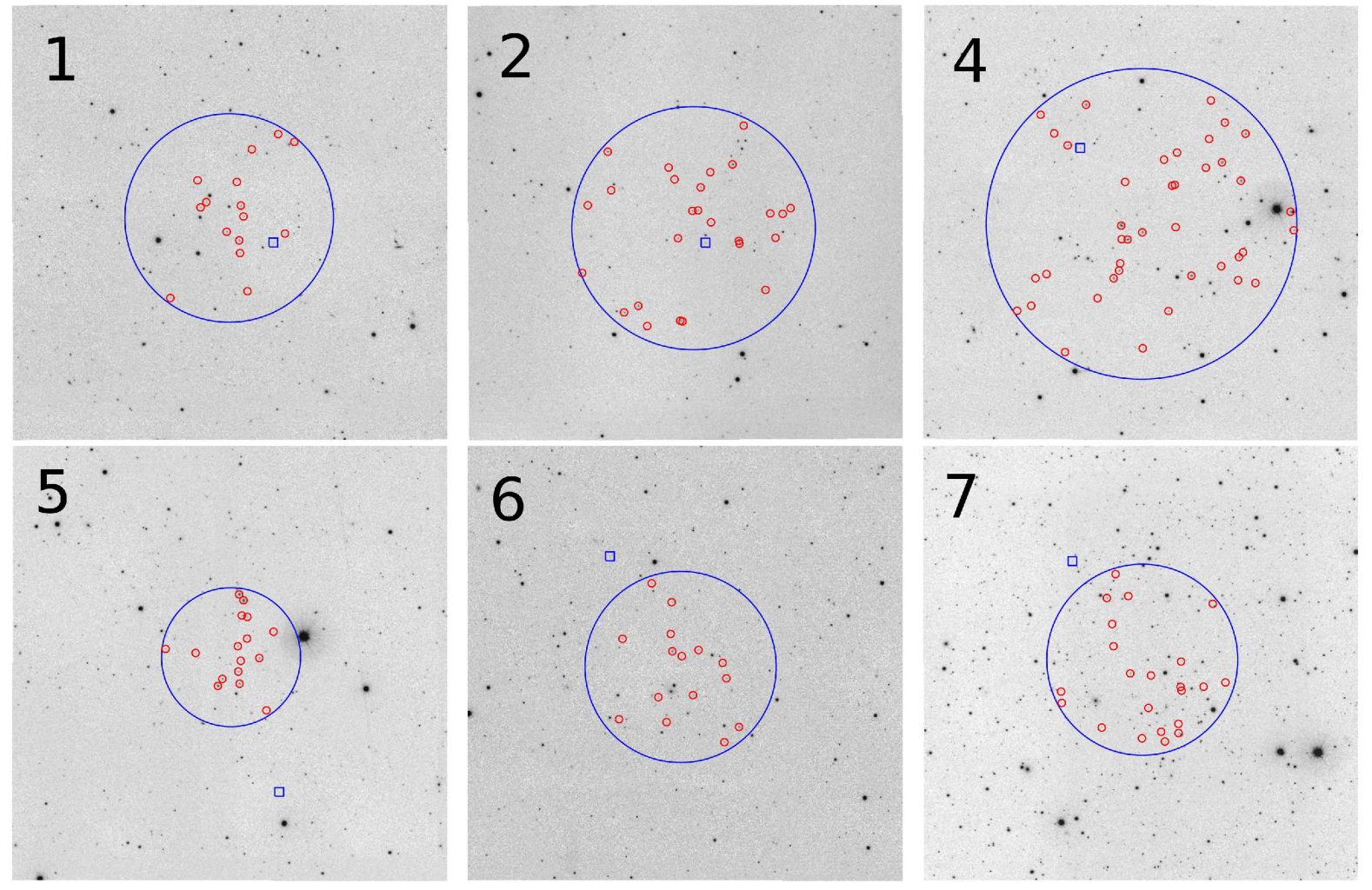

Figure 9. $J$-band images of 6 galaxy clusters identified near our SMG candidates (labelled with the corresponding number on each image). Blue circles mark the core of the cluster. Red circles mark galaxies in the red sequence of the cluster. Blue boxes mark the positions of SMG candidates.

\section{REFERENCES}

Blain, A. W., 1996 MNRAS 283, 1340

Bothwell, M. S. et al. 2013, MNRAS, 429, 3047

Bèthermin, M. et al., 2015, A\&A, 573, 113

Bussmann, R. S. et al. 2013, ApJ 779,25

Calanog, J. A., et al. 2014, ApJ, 797, 138

Cardelli, Jason A., Clayton, Geoffrey C. and Mathis, John S., 1989, ApJ 345, 245.

Casey, C. M., Narayanan, D., \& Cooray, A. 2014, PhR, 541,45

Cañameras et al. 2015, A\&A, 581, 105

Chapman, S. C., Blain, A. W., Smail, Ian \& Ivison, R. J., 2005, ApJ 622, 772

Chen, C.-C., et al.,2016, ApJ,820, 82

Cohn, J.D. et al., 2007, MNRAS, 382, 1738

Condon,J.J.,1992,ARA\&A,30,575

Dalton, G. B., Caldwell, M., Ward, A. K., et al. 2006 SPIE, 6269, 62690

Danielson, A. et al. 2011, MNRAS, 410, 1687

Dannerbauer, H., Lehnert, M. D., Lutz, D., et al. 2002,

ApJ, 573,473

Dannerbauer, H. et al. 2008, ApJl, 673, 123

Dunlop, J. et al. 2004, MNRAS, 350, 769

Dye, S. et al.,2015 MNRAS 452, 2258

Emerson, J.P 2001, ASPC 232, 339E

Emerson, J.P et al. 2004, SPIE.5,493, 401E

Gladders, M.D., and Yee, H.K., 2000, AJ, 120, 2148

Gonzalez, Anthony H., Papovich, Casey, Bradac, Marusa,
Jones, Christine, 2011, ApJ 720, 245

Greve, T. R. et al. 2005, MNRAS, 359, 1165

Harrington, K. C. et al, 2016, MNRAS 458, 4383

Hayward, C. C., Narayanan, D. „Kereŝ, Dusân, Jonsson, Patrik, Hopkins, Philip F., Cox, T. J.\& Hernquist, L. 2013, MNRAS 428, 2529

Hodge, J. A. et al. 2015, ApJL, 798, 18

Hodge, et al. 2016, ApJ, in press (astro-ph/1609

Hughes, D. H. et al., 1998, Nature, 394, 241

Ikarashi, S. et al. 2015, ApJ, 860, 133

Irwin, M. J., Lewis, J., Hodgkin, S., Bunclark, P., Evans, D., McMahon, R., Emerson, J. P., Stewart, M.\& Beard, S., 2004, SPIE 5493, 411

Ivison R. J. et al., 2010, A\&A, 518, L35

Ivison, R. et al. 2013, ApJ, 772, 137

Johansson, D. et al. 2011, A\&A, 527, 117

Karim, A. et al. 2013, MNRAS, 432, 2

Lapi, A. et al., 2011, ApJ 742, 24L

Lewis, J. R., Irwin, M. J. \& Bunclark, P. S. 2010, ASP Conf. Ser., 434, 91

Ma, B., et al. 2015, ApJ, 814, 17

Ma, J., et al. 2015, ApJ, 812, 88

Magnelli, B. et al. 2012, A\&A, 539, 155

Messias, H., et al. 2014, A\&A, 568, 92

Michalowski, M. J.,Dunlop, J. S., Cirasuolo, M., Hjorth, J., Hayward, C. C.\& Watson, D, 2012, A\&A, 541, 85

Miettinen, O. et al. 2015, A\&A, 577, 29

Narayanan, D. et al. 2015, Nature, 529, 496

Negrello, M., Perrotta, F., Gonźalez-Nuevo, J., Silva, L.,de 
Zotti, G., Granato, G.L., Baccigalupi, C., Danese, L.,2007, MNRAS, 277, 1557

Negrello, M. et al., 2010, Science, 330, 800

Perrotta, F., Baccigalupi, C., Bartelmann, M., De Zotti, G. \& Granato, G. L., 2002 MNRAS 329, 445

Perrotta, F., Magliocchetti, M., Baccigalupi, C., Bartelmann, M., De Zotti, G., Granato, G. L., Silva, L. \& Danese, L., 2003, MNRAS 338, 623

Polletta, M. et al. 2007, ApJ, 663, 81

Pope, A. et al. 2008, ApJ, 675, 1171

Schlegel, D. J., Finkbeiner, D. P. \& Davis, M., 1998, ApJ, 500,525

Simpson, J. M. et al. 2014, ApJ, 788, 125

Simpson, J. M. et al. 2015, ApJ, 799, 81

Smail, Ian, Ivison, R. J. \& Blain, A. W., 1997, ApJ 490, L5

Smail, I., Ivison, R. J., Blain, A. W. \& Kneib, J.-P., et al. 2002, MNRAS, 331, 495

Spilker, J. S et al., 2016, ApJ, 826,112

Strandet, M. L. et al. 2016, ApJ, 822, 80

Swinbank A. M. et al., 2008, MNRAS 391, 420

Swinbank A. M. et al., 2010, Nature, 464, 733

Swinbank, A. M., et al. 2015, ApJL, 806, 17

Tonini et al., 2012, ApJ, 759, 43

Vieira J. D. et al., 2010, ApJ, 719, 763

Vieira, J., et al. 2013, Nature, 495, 344

Younger, J. D. et al. 2007, ApJ, 671, 1531

Walter, F., et al. 2012, Nature, 486, 233

Weiss, A. et al., 2013, ApJ, 767, 88

Wright, E. L. et al., 2010, ApJ 140, 1868-1881 